\title{
Harvesting the Electromagnetic Energy Confined Close to a Hot Body
}

https://doi.org/10.1515/zna-2019-0132

Received April 24, 2019; accepted May 1, 2019; previously

published online May 27, 2019

\begin{abstract}
In the close vicinity of a hot body, at distances smaller than the thermal wavelength, a high electromagnetic energy density exists due to the presence of evanescent fields radiated by the partial charges in thermal motion around its surface. This energy density can surpass the energy density in vacuum by several orders of magnitude. By approaching a photovoltaic (PV) cell with a band gap in the infrared frequency range, this nonradiative energy can be transferred to it by photon tunnelling and surface mode coupling. Here we review the basic ideas and recent progress in near-field energy harvesting.
\end{abstract}

Keywords: Energy Conversion; Nanoscale Heat Transfer; Near-Field.

\section{Introduction}

Thermophotovoltaic devices [1, 2] are energy conversion systems that generate electric power directly from thermal radiation. While in classical photovoltaic conversion devices the efficiency (defined as the ratio of the electric power produced by the cell over the net radiative power exchanged between the primary source and the cell) is bounded by the thermodynamic Shockley-Queisser limit [3] (30\% for cells with a gap energy $E_{g}=1 \mathrm{eV}$ and $10 \%$ for $E_{g}=0.5 \mathrm{eV}$ ), with near-field thermophotovoltaic (NTPV) systems this limit, in principle, can be surpassed by exploiting the strong heat transfer due to tunnelling of nonpropagating photons. In particular, with a quasimonochromatic source, as those made with materials that support a surface wave, the efficiency can be close to $35 \%$ or even higher when this frequency coincides with the gap frequency of semiconductor [4]. Moreover, the strong magnitude of exchanges in plane-plane geometry [5] in near-field regime compared with what happens in far-field

*Corresponding author: Philippe Ben-Abdallah, Laboratoire Charles Fabry, UMR 8501, Institut d'Optique, CNRS, Université Paris-Sud 11, 2, Avenue Augustin Fresnel, 91127 Palaiseau Cedex, France, E-mail: pba@institutoptique.fr

Svend-Age Biehs: Institut für Physik, Carl von Ossietzky Universität, D-26111 Oldenburg, Germany, E-mail: s.age.biehs@uol.de regime (which cannot exceed the blackbody limit [6]) can lead to a generated power between 1 and $120 \mathrm{~W} \mathrm{~cm}^{-2}$ in the temperature range $500-1200 \mathrm{~K}$ for separation distances between the source and the cell of $10 \mathrm{~nm}$ [7]. As a result, a cell of $25 \mathrm{~cm}^{2}$ could theoretically generate a power of 25-3000 W. This is a very large value. The typical energy demand of a household in the United States [8] is $2500 \mathrm{~W}$, for instance.

In this article, we describe the basic principles behind this near-field technology, and we briefly discuss the main issues that limit to date its massive deployment.

\section{Electromagnetic Energy Close to a Hot Body}

It is well known that the spectral energy density of thermal radiation at a given equilibrium temperature $T$ follows the Planck law:

$$
u_{\mathrm{BB}, \omega}=\Theta(T) D_{\mathrm{vac}}(\omega)
$$

where

$$
\Theta(T)=\frac{\hbar \omega}{\mathrm{e}^{\hbar \omega / k_{\mathrm{B}} T}-1}
$$

is the mean energy of a harmonic oscillator in thermal equilibrium and

$$
D_{\mathrm{vac}}(\omega)=\frac{\omega^{2}}{\pi^{2} c^{3}}
$$

is the density of states in vacuum. Here, we have introduced the Boltzmann constant $k_{\mathrm{B}}$, the reduced Planck constant $\hbar$, and the light velocity in vacuum $c$. The maximum of the Planck spectrum is at a frequency of $\omega_{\text {th }}=2.82 \omega_{c}$ with $\omega_{c}=k_{\mathrm{B}} T / \hbar$. As function of wavelength, the maximum is at the thermal wavelength $\lambda_{\text {th }}$ given by Wien's displacement law $\lambda_{\text {th }}=2897 \mu \mathrm{mK} / T$, i.e. at $300 \mathrm{~K}$, the dominant frequency is $\omega_{\text {th }} \approx 10^{14} \mathrm{rad} / \mathrm{s}$ and the dominant wavelength $\lambda_{\text {th }} \approx 10 \mu \mathrm{m}$.

Now, close to a thermal emitter at distances smaller than the thermal wavelength $\lambda_{\text {th }}$, the properties of the emitter itself enter into the density of states and therefore also into Planck's law. Close to a thermal emitter as sketched in Figure 1 in the particular case of a hexagonal 


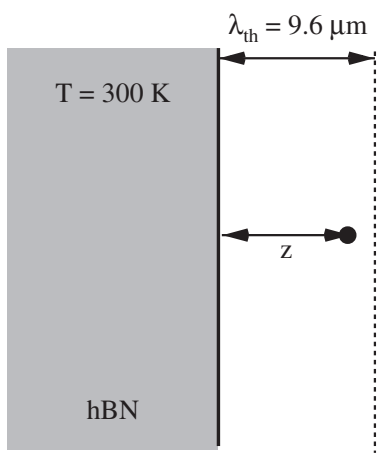

Figure 1: Sketch of the planar sample in global equilibrium at temperature $T=300 \mathrm{~K}$. The energy density at a near-field distance $z<\lambda_{\text {th }}=9.6 \mu \mathrm{m}$ highly depends on the properties of the thermal emitter, which here is hBN.

boron nitride (hBN) sample, a generalised Planck law can be derived, which has the form [9-11]:

$$
u_{\mathrm{th}, \omega}=\Theta(T) D_{\mathrm{loc}}(\omega)
$$

where $D_{\text {loc }}(\omega)$ is the local density of states. It depends in general on the geometry and optical properties of the emitter and on the position with respect to the emitter at which it is evaluated. For an emitter with a planar interface due to the translation symmetry with respect to planes parallel to the interface, it depends on the distance $z$ and the properties of the emitter. It can be shown that for $z \gg \lambda_{\text {th }}$ it converges to the density of states in vacuum $D_{\text {loc }} \rightarrow D_{\text {vac }}$, and in the opposite quasi-static regime for $z \ll \lambda_{\text {th }}$, it becomes larger than the vacuum density of states and diverges like $D_{\text {loc }} \propto 1 / z^{3}$. Therefore, the energy density of thermal radiation close to a hot body is in general distance dependent, and its magnitude can be larger than that predicted by Planck's law. This can be attributed to the contribution of evanescent waves existing in the vicinity of the emitter, which have electromagnetic fields that are exponentially decaying with respect to the emitters interface. Such waves are, for example, total internal reflection waves and surface waves. As was already shown by Eckhardt [10] and later studied in detail by Shchegrov et al. [12] and Joulain et al. [13], the surface waves can lead to an extremely enhanced quasi-monochromatic near-field energy density spectrum. In metals, such surface waves are surface plasmon-polaritons, and in polar dielectrics, such surface waves are known as surface phonon-polaritons (SPhP).

In Figure 2, we show the energy density close to an $\mathrm{hBN}$ emitter. The optical phononic properties of hBN are described by the Drude-Lorentz model [14]:

$$
\varepsilon_{\mathrm{e}}(\omega)=\varepsilon_{\infty} \frac{\omega^{2}-\omega_{\mathrm{L}}^{2}+i \gamma \omega}{\omega^{2}-\omega_{\mathrm{R}}^{2}+i \gamma \omega},
$$

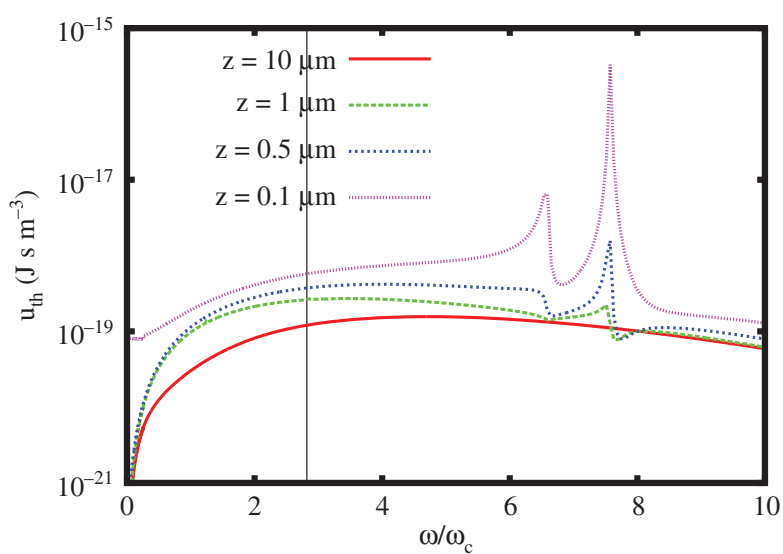

Figure 2: Semi-log plot of the spectral energy density $u_{\mathrm{th}, \omega}$ in a distance $z$ above an hBN half-space at $T=300 \mathrm{~K}$. The thermal wavelength is therefore $10 \mu \mathrm{m}$. Therefore, the spectrum at $z=10 \mu \mathrm{m}$ coincides mainly with the blackbody spectrum $u_{\mathrm{BB}, \omega}$. The vertical line is at the maximum frequency of the blackbody spectrum at $2.82 \omega_{\mathrm{c}}$ with $\omega_{\mathrm{c}}=3.5 \times 10^{13} \mathrm{rad} / \mathrm{s}$. For more details, see [11-13].

with $\varepsilon_{\infty}=4.88, \omega_{\mathrm{L}}=3.032 \times 10^{14} \mathrm{rad} / \mathrm{s}, \omega_{\mathrm{R}}=2.575 \times$ $10^{14} \mathrm{rad} / \mathrm{s}$, and $\gamma=1.001 \times 10^{12} \mathrm{rad} / \mathrm{s}$. As shown in Figure 2, for hBN, which supports SPhP, the spectral energy density becomes dominated by the $\mathrm{SPhP}$ resonance at the frequency $\omega_{\text {sp }}$ for very small distances $z \ll \lambda_{\text {th }}$. This resonance frequency is in general for surface modes on planar interfaces implicitly defined by the condition $\operatorname{Re}\left(\varepsilon\left(\omega_{\mathrm{sp}}\right)\right)=-1$ where $\varepsilon$ is the complex permittivity of the emitter, which here has an interface with the vacuum. For metals, the surface mode resonances are typically in the UV region, and for polar dielectrics, they are in the infrared. In particular, for hBN, the resonance frequency is $\omega_{\mathrm{sp}}=2.960 \times 10^{14} \mathrm{rad} / \mathrm{s}$, which corresponds to a wavelength of $5 \mu \mathrm{m}$. Hence, the resonance is very close to the thermal wavelength $\lambda_{\text {th }}$ at $300 \mathrm{~K}$ and can therefore be thermally excited at room temperature, which is not the case for the surface waves in metals. Note that the thermal nearfield energy density can be tuned by texturing the material or by deposing 2D sheets on its surface [15, 16], and it is accessible with different scanning probe devices [17-23].

\section{Near-Field Heat Transfer}

When bringing a receiver with temperature $T_{\mathrm{r}}$ close to an emitter at higher temperature $T_{\mathrm{e}}>T_{\mathrm{r}}$ as sketched in Figure 3, then in general the propagating and evanescent waves generated by the thermal motion of the charges inside the emitter and receiver will contribute to the heat transfer. It has already been shown in the 1970s that when the receiver enters the near-field regime of the emitter, i.e. for emitter-receiver distances $d<\lambda_{\text {th }}$, then the resulting 


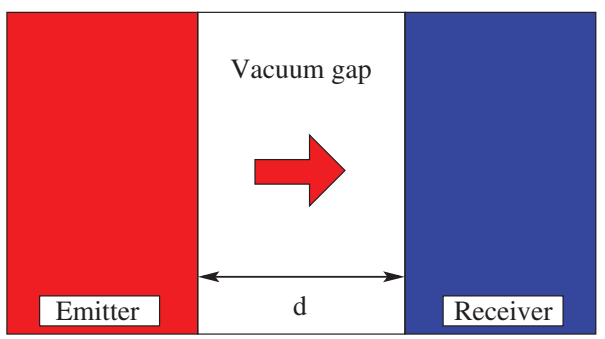

Figure 3: Sketch of the emitter and receiver separated by a vacuum gap of thickness $d$. In NTPV, the receiver is replaced by a low band-gap photovoltaic cell.

radiative heat flux $\Phi$ in this case can become much larger than the blackbody value due to the contribution of the evanescent waves, which are confined at the surface of the emitter when the distance becomes smaller than the dominant thermal wavelength [5, 24-26]. About 30 years later, the idea to exploit the huge energy density of the evanescent waves for energy harvesting was brought forward by replacing the receiver by a photovoltaic cell [2730]. In [28], a first detailed study of near-field thermophotovoltaics (NTPV) cells based on the framework of fluctuational electrodynamics [31] is provided. A first experimental proof of concept was conducted by DiMatteo et al. [32]. These works paved the way for a large number of mainly theoretical works on NTPV.

The theoretical foundation is the general expression for the heat flux between two materials with planar interfaces, which was first determined within the framework of fluctuational electrodynamics [31] by Polder and van Hove [5]. This expression can be cast into a Landauer-like form by $[33,34]$ :

$$
\Phi(d)=\int_{0}^{\infty} \frac{d \omega}{2 \pi} \Delta \Theta(\omega) \sum_{j=s, \mathrm{p}} \int \frac{d^{2} \mathbf{k}_{\perp}}{(2 \pi)^{2}} \mathcal{T}_{j}\left(\omega, \mathbf{k}_{\perp} ; d\right)
$$

where

$$
\Delta \Theta(\omega)=\Theta\left(T_{\mathrm{e}}\right)-\Theta\left(T_{\mathrm{r}}\right)
$$

and $\mathcal{T}_{j}\left(\omega, \mathbf{k}_{\perp} ; d\right) \in[0: 1]$ are the transmission factor for a given transversal mode with lateral wave vector $\mathbf{k}_{\perp}=$ $\left(k_{x}, k_{y}\right)^{t}$, frequency $\omega$, and polarisation $j$ (s - TE polarisation; $\mathrm{p}$ - TM polarisation). The transmission factor $\mathcal{T}_{j}\left(\omega, \mathbf{k}_{\perp} ; d\right)$ is fully determined by the optical properties of the receiver and emitter. The contributions of the propagating waves are restricted to waves with wave vectors $\left|\mathbf{k}_{\perp}\right| \leqslant k_{0}$ and the evanescent wave contributions stem from waves with wave vectors $\left|\mathbf{k}_{\perp}\right|>k_{0}$, where $k_{0}=\omega / c$ is the wave number in vacuum.

It is clear that the maximum contribution of the propagating waves is obtained by setting $\mathcal{T}_{j} \equiv 1$ for all $\left|\mathbf{k}_{\perp}\right| \leqslant k_{0}$. Then the above expression gives the blackbody result:

$$
\Phi_{\mathrm{BB}}=\sigma_{\mathrm{BB}}\left[T_{\mathrm{e}}^{4}-T_{\mathrm{r}}^{4}\right]
$$

where $\sigma_{\mathrm{BB}}=5.67 \times 10^{-8} \mathrm{Wm}^{-2} \mathrm{~T}^{-4}$ is the StefanBoltzmann constant. As a consequence, it becomes apparent that the contribution of the evanescent waves will add to the heat flux of the propagating waves, and therefore it can be larger than $\Phi_{\mathrm{BB}}$. In Figure 4, the contributions of the different heat flux channels for two hBN plates are highlighted. It can be seen that the propagating modes dominate in the far-field regime with $d>\lambda_{\text {th }}$, whereas the evanescent modes dominate in the near-field regime with $d<\lambda_{\text {th }}$. The near-field regime can be divided into the regime $d<\lambda_{\mathrm{th}} / 100$, where the surface modes dominate, and the intermediate regime with $\lambda_{\text {th }} / 100<d<$ $\lambda_{\text {th }}$, where the frustrated modes dominate [35-37]. The behaviour observed in Figure 4 is similar for other materials like $\mathrm{SiC}$, silica, or sapphire supporting surface modes in the infrared. For example, for $\mathrm{SiC}$ with a surface mode resonance at $\omega_{\mathrm{sp}}=1.787 \times 10^{14} \mathrm{rad} / \mathrm{s}$, the enhancement would be $1000 \Phi_{\mathrm{BB}}$ at $d=10 \mathrm{~nm}$ for the choice of temperatures in Figure 4, and for silica, even higher enhancement factors are achievable. Depending on the shape and temperature regimes, optimised material parameters can be obtained to increase the energy density and the heat flux [38-40].

First measurements of the near-field heat transfer between two planar samples were already conducted in the 1970s by Cravalho et al. [41], Hargreaves [42],

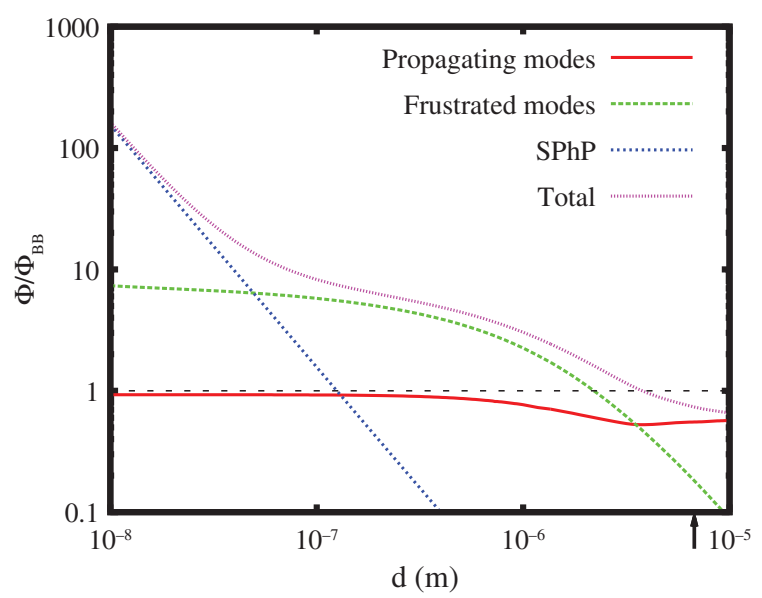

Figure 4: Near-field heat flux $\Phi$ between two hBN half-spaces normalised to the blackbody value $\Phi_{\mathrm{BB}}$ as function of distance $d$. Here, the temperature of the emitter is $300 \mathrm{~K}$, and the temperature of the receiver is $0 \mathrm{~K}$ so that $\Phi_{\mathrm{BB}}=459.27 \mathrm{Wm}^{-2}=0.046 \mathrm{Wcm}^{-2}$. The contribution of different modes to the near-field heat flux is highlighted. For more details, see [35]. 
and Domoto et al. [43] for metallic samples. A new era of near-field measurements has been started with the seminal work of Ottens et al. [44] in 2011, who could measure the heat flux between macroscopic $50 \times 50 \mathrm{~mm}$ sapphire plates, which support surface modes in the infrared, down to a distance of about $2 \mu \mathrm{m}$. The aspect ratio between the smallest interplate distance to the lateral extension of the samples is approximately 1:25,000. The measured enhancement was found to be 1.26 times the blackbody value [44] and can be attributed to the contribution of the frustrated total reflection modes. The following works have improved the experimental techniques in order to reduce the interplate distances as much as possible and to achieve the highest possible near-field enhancement [44-57]. State-of-the-art setups like the experiment by Fiorino et al. [57] are now capable of measuring the heat flux between a $50 \times 50 \mu \mathrm{m}$ silica emitter and an extended silica receiver down to $25 \mathrm{~nm}$ and found an enhancement of about 700 times the blackbody value proving the possibility to have extremely large radiative heat fluxes due to the surface mode interaction in silica. Note that in most recent experimental setups the smaller distances are achieved by reducing the size of the samples so that, for example, the aspect ratio in the experiment [57] is 1:2000.

One of the technical challenges for near-field thermophovoltaics will be to use the near-field effect for largescale PV cells. To achieve this goal, several structures using different kinds of spacers having a small heat conductivity were proposed [45, 50, 53, 54]. The spacers make it possible to keep the emitter and receiver in a controlled near-field distance. For example, Ito et al. [50] measured in such a structure the radiative heat flux between two $19 \times 8.6 \mathrm{~mm}$ quartz plates with microstructured pillars down to $500 \mathrm{~nm}$ [50]; i.e. the aspect ratio is in this case about 1:17,200. One of the drawbacks of such a solution is that the heat conduction through the pillars is relatively large. Consequently, by this conduction, the receiver will heat up, which will reduce the efficiency of any NTPV device. In the setup of Ito et al. [50], the heat flux through the spacers was on the same order of magnitude as the radiative heat flux between the emitter and the receiver. The goal of future setups or devices using spacers is therefore clearly to reduce this contribution of heat conduction through the spacers while having large areas for thermal radiation and a high stability.

\section{Near-Field Energy Conversion}

As demonstrated in the previous sections, the radiative heat flux by the surface phonon-polariton contribution can be extremely large, and as the SPhP contribution to the energy density, it can also be quasi-monochromatic. This is a promising feature for exploitation in NTPV devices. It has been shown in a theoretical study by Narayanaswamy and Chen [58] in 2003 that the surface modes of phononpolaritonic emitters like SiC, cubic, and hBN will result in a tremendous increase of the heat flux into a direct band gap PV cell. A quantitative calculation of the photocurrent and electric power in a tungsten-GaSb configuration was provided by Laroche et al. [4] assuming $100 \%$ quantum efficiency of the PV cell; i.e. it is assumed that all the incoming or absorbed heat radiation will be converted into a photocurrent. It could be shown that the conversion efficiency converges in this case towards $29 \%$ for distances below $100 \mathrm{~nm}$. Theoretically, a generated electric power output of $3 \times 10^{4} \mathrm{~W} \mathrm{~m}^{-2}$ was reported for a distance of $5 \mathrm{~nm}$. It should be noted that the Shockley-Queisser limit for a blackbody illuminating a GaSb cell is an efficiency of $29 \%$. It was theoretically demonstrated that a quasi-monochromatic emitter like those considered by Narayanaswamy and Chen [58] can even beat the Shockley-Queisser limit in the near-field regime yielding $35 \%$ at an extremely small distance of $5 \mathrm{~nm}$, which can be hardly achieved in real NTPV devices.

To illustrate the potential of the NTPV technology for energy harvesting, we consider a system composed by a hot emitter made of hBN at temperature $T_{\mathrm{e}}$ placed in the proximity of a junction cell at temperature $T_{\mathrm{r}}<T_{\mathrm{e}}$ made of indium antimonide (InSb) with a gap frequency $\omega_{g}=$ $1.8231 \times 10^{14} \mathrm{rad} / \mathrm{s}\left(E_{g}=0.12 \mathrm{eV}\right)$. We use the optical properties of hBN from (5) and the dielectric function of the PN junction defined as follows [59]:

$$
\varepsilon_{\mathrm{r}}(\omega)=\left(\begin{array}{cl}
n_{r}^{2}(\omega), & \omega<\omega_{g}, \\
\left(n_{r}(\omega)+\mathrm{i} n_{i}(\omega)\right)^{2}, & \omega>\omega_{g},
\end{array}\right.
$$

where

$$
n_{r}=\sum_{j=1}^{4} \frac{B_{j} \omega+C_{j}}{\omega^{2}-B_{j} \omega+C_{j}}
$$

and

$$
n_{i}=\frac{\alpha_{0} c}{2 \omega} \sqrt{\frac{\omega}{\omega_{g}}-1}
$$

with $B_{j}=\frac{a_{j}}{q_{j}}\left(E_{g} b_{j}-\frac{1}{2} b_{j}^{2}-E_{g}^{2}+c_{j}\right), C_{j}=\frac{a_{j}}{q_{j}}\left(\left(E_{g}^{2}+c_{j}\right) \frac{b_{j}}{2}-\right.$ $\left.2 E_{g} c_{j}\right)$ and $q_{j}=\frac{1}{2} \sqrt{4 c_{j}-b_{j}^{2}}$. The constants $a_{j}, b_{j}$, and $c_{j}$ $(j=1-4)$ are tabulated values and dependent on the 
type of semiconductors [59]. It is direct to verify that hBN supports an SPhP resonance at frequency $\omega_{\text {sp }} \simeq 2.960 \times$ $10^{14} \mathrm{rads}^{-1}$, larger than $\omega_{\mathrm{g}}$ as desired.

The radiative power exchanged between the source and the junction reads

$$
\begin{aligned}
& P_{\mathrm{rad}}=\int_{0}^{\infty} \frac{d \omega}{2 \pi} K(\omega)\left[\Theta\left(\omega, T_{\mathrm{e}}\right)\right. \\
&\left.-\Theta\left(\omega-\omega_{0}, T_{\mathrm{r}}\right) H\left(\omega-\omega_{\mathrm{g}}\right)\right] .
\end{aligned}
$$

where $H$ denotes the Heaviside function, $\omega_{0}=e V_{0} / \hbar . V_{0}$ being the potential difference at which the cell is operating and which is here [60] taken as $\hbar \omega_{g}\left(1-T_{\mathrm{r}} / T_{\mathrm{e}}\right) / e$ introducing the elementary charge $e$. The quantity

$$
K(\omega)=\sum_{j=s, \mathrm{p}} \int \frac{\mathbf{d}^{2} \mathbf{k}_{\perp}}{(2 \pi)^{2}} \mathcal{T}_{j}\left(\omega, \mathbf{k}_{\perp} ; d\right)
$$

is the number of modes per unit area that contribute to the transfer weighted by the Landauer transmission probability $\mathcal{T}_{j}(\omega, k)$ between the source and the cell in polarisation $j$ of the energy $\hbar \omega$ carried by the mode $\left(\omega, \mathbf{k}_{\perp}\right)$. The two terms in expression (12) represent the emission radiated by the source toward the cell and the power radiated back from the cell in direction of the source, respectively. As for the electric power density, which is generated in the cell, it reads

$$
P_{\mathrm{PV}}=e V_{0} \int_{\omega_{g}}^{\infty} \frac{d \omega}{2 \pi}\left[\Theta\left(\omega, T_{\mathrm{e}}\right)-\Theta\left(\omega-\omega_{0}, T_{\mathrm{r}}\right)\right] \frac{K(\omega)}{\hbar \omega}
$$

assuming a quantum efficiency of $100 \%$. In Figure 5, we show the evolution of the power transferred to the cell and the electric power generated by the system with respect to the separation distance $d$ between the source and the cell. We also plot in Figure $5 \mathrm{~b}$ the efficiency $\eta=P_{\mathrm{PV}} / P_{\mathrm{rad}}$ of the cell. For separation distances larger than the thermal wavelength $\lambda_{\text {th }}$, the power exchanged between the source and the cell is mainly due to propagating photons so that it is independent of the distance. At subwavelength distances, the radiative heat exchanges increase as the separation distance decays. This enhancement of exchanges is directly related to the number of contributing modes with respect to the separation distance [33, 34]. As discussed in the previous section at subwavelength distance, the nonpropagating modes (i.e. evanescent modes) superimpose to the propagating ones, giving rise to new channels for heat exchanges by photon tunnelling between the source and the cell. As a direct consequence, these photons can generate further electron-hole pairs inside the junction and therefore increase the production of electricity. Hence, at $d=500 \mathrm{~nm}$, the generated power is doubled compared
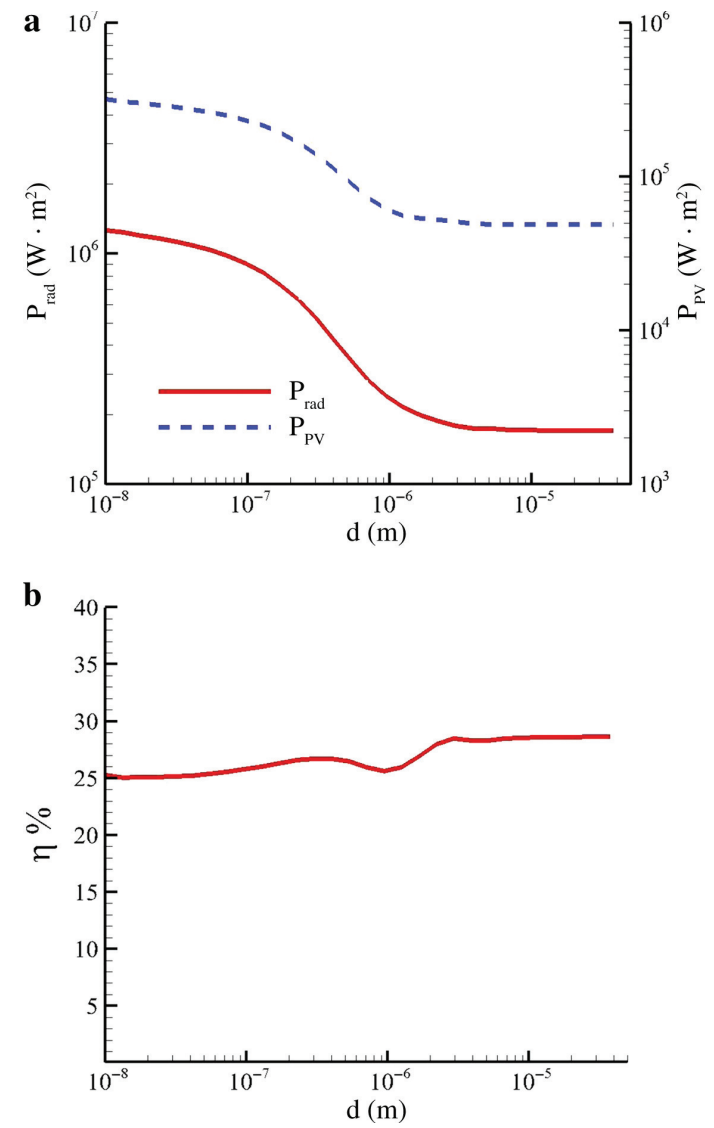

Figure 5: (a) Radiative power exchanged between an hBN thermal source at $T_{\mathrm{e}}=1500 \mathrm{~K}$ and an $\ln \mathrm{Sb}$ junction at $T_{\mathrm{s}}=300 \mathrm{~K}$ and electric power generated in this system with respect to the separation distance. (b) Efficiency of produced electricity in an hBN/InSb TPV conversion system with respect to the separation distance between the source and the cell.

to what happens in far-field regime. In extreme near-field regime, this power is almost an order of magnitude larger than in far-field regime. As the efficiency is concerned, we see in Figure $5 b$ that in near-field regime the efficiency is a bit reduced in comparison with the performances of TPV conversion device in far-field regime. Nevertheless, we have in this case an efficiency of about $25 \%$, and the output power at a realistic distance of $100 \mathrm{~nm}$ is approximately $220 \mathrm{~kW} \mathrm{~m}^{-2}$. Note that while the $\mathrm{SPhP}$ of hBN is located at $5 \mu \mathrm{m}$, the gap of InSb cell is around $7.3 \mu \mathrm{m}$. This spectral mismatch reduces the conversion performances. By adding a sheet of graphene to the emitter or receiver, the heat flux by the surface modes and hence the efficiency can be increased in the extreme near-field regime $[7,60,61]$. In [7], an efficiency of $30 \%(40 \%)$ with a generated power of $6 \mathrm{~W} \mathrm{~cm}{ }^{-2}\left(120 \mathrm{~W} \mathrm{~cm}^{-2}\right)$ was reported for an emitter temperature of $600 \mathrm{~K}(1200 \mathrm{~K})$ at a distance of $10 \mathrm{~nm}$. 


\section{State of the Art}

By using a much more realistic model of the PV cell, Park et al. [62] have shown that in a tungsten-InGaSb configuration the efficiency can substantially decrease when making the distance between the emitter and the PV cell smaller. This is due to the fact that the penetration depth of the evanescent waves inside the PV cell becomes very small, because the evanescent waves are exponentially damped on a distance on the order of the distance $d$ in the quasi-static regime. For surface modes, it could be shown [63] that the penetration depth scales like 0.25 $d$ for phonon-polaritonic materials like $\mathrm{SiC}$. As a consequence, for very small distances, the heat flux increases dramatically due to the evanescent wave contributions, but it will be dissipated at the interface mainly. Hence, only electron-hole pairs close to the interface of the PV cell will be generated, limiting such the conversion efficiency. Park et al. [62] report an efficiency of about $20 \%$ at a distance of $10 \mathrm{~nm}$. Nonetheless, the theoretically predicted generated electric power is still high and has a nominal value of $10^{6} \mathrm{~W} \mathrm{~m}^{2}$. Introducing a back reflector to the PV cell could even improve the performance [8]. A detailed discussion of the contribution of the different heat flux channels to the NTPV efficiency can be found in [64].

Since the work of Park et al., the modelling has been further improved, and the Moss-Burnstein effect, the impact of series resistance, photon recycling, and parasitic sub-band-gap absorption and cell cooling, has been studied in detail [65-69]. Furthermore, hyperbolic materials have been proposed for replacing the emitter, receiver, or the gap region [70-73], because with these materials, spectral control, high heat flux levels, and large penetration depths are achievable [74-78]. For example, with the NTPV system in [73] using a $\mathrm{W} / \mathrm{SiO}_{2}$ hyperbolic emitter and an InAs cell, a generated power of $1.78 \mathrm{kWm}^{-2}$ is reported at a realistic distance of $100 \mathrm{~nm}$.

Despite all those efforts on the theoretical side, there is only a single up-to-date experimental study of an NTPV system conducted by Fiorino et al. [79]. In this pioneer experimental study, a Si emitter at temperatures ranging from 525 to $665 \mathrm{~K}$ is brought in close vicinity of two different TPV cells with band gaps of 0.345 and $0.303 \mathrm{eV}$. The measured power generated at the high (small) bandgap cell at a nominal distance of $60 \mathrm{~nm}$ is 40 times larger than at long separation distances. However, this power remains relatively small (around $30 \mathrm{nW}$ for $T_{\mathrm{e}}=655 \mathrm{~K}$ ) because heat is essentially transferred with frustrated photons. Taking into account the area of circular emitter of radius of $40 \mu \mathrm{m}$, we obtain a power flux of about $6 \mathrm{~W} \mathrm{~m}^{-2}$ and an aspect ratio of 1:3200. Although this value is relatively small and corresponds to an extremely low conversion efficiency $(\approx 0.02 \%)$ several points can significantly be improved. In particular, by scaling up the system size and increasing the emitter temperature $1000 \mathrm{~K}$, a $6 \%$ conversion efficiency can be expected. Also, using an emitter that supports a surface wave in the Planck window, the radiative heat exchanges between the emitter and the PV cell could surpass by several orders of magnitude the flux exchanged at long separation distances. With these improvements, the efficiency of NTPV systems could be even larger than the Schockley-Queisser limit [3].

\section{Concluding Remarks}

In conclusion, the basic concepts to convert the near-field electromagnetic energy confined close to a hot body into electricity have been introduced. We have shown that this near-field technology allows to largely surpass the performances of the classical TPV technology, despite that its potential several hurdles strongly limit to date the development and the massive deployment of this technology. On the one hand, near-field heat flux experiments between planar structures in the near-field regime are nowadays possible even down to $25 \mathrm{~nm}$ distance, but typically not for systems with large areas. Using low conducting spacers to keep the vacuum gap in the system is one possible workaround. Another is to replace the vacuum gap by a low-conducting but high-index or hyperbolic material as recently proposed [72]. Furthermore, it is not trivial to maintain large temperature gradients over very small gaps, and the cooling of the cell could further reduce the efficiency of the cell, because the power used to cool the cell has to be taken into account in a global balance. So far, a power output of only $6 \mathrm{~W} \mathrm{~m}^{-2}$ and an efficiency of only $0.02 \%$ seem to be a little bit disappointing, but the NTPV technology has a great potential, and we are very optimistic that future theoretical and experimental works will improve the efficiencies of NTPV systems step by step to a point where the promised near-field enhancement will be achieved and power outputs of $1 \mathrm{~kW} \mathrm{~m}^{-2}-1 \mathrm{MW} \mathrm{m}^{-2}$ and efficiencies around $20 \%-40 \%$ are not just the dream of theorists but reality.

Acknowledgments: S.-A. B. acknowledges support from Heisenberg Programme of the Deutsche Forschungsgemeinschaft (DFG, German Research Foundation) under the project no. 404073166 . 


\section{References}

[1] T. J. Coutts, Renew. Sust. Energ. Rev. 3, 77 (1999).

[2] A. Lenert, D. M. Bierman, Y. Nam, W. R. Chan, I. Celanovic, et al., Nat. Nanotechnol. 9, 126 (2014).

[3] W. Shockley and H. Queisser, J. Appl. Phys. 32, 510 (1961).

[4] M. Laroche, R. Carminati, and J.-J. Greffet, J. Appl. Phys. 100, 063704 (2006).

[5] D. Polder and M. van Hove, Phys. Rev. B 4, 3303 (1971).

[6] M. Planck, The Theory of Heat Radiation, Forgotten Books, Leipzig 2010.

[7] O. Ilic, M. Jablan, J. D. Joannopoulos, I. Celanovic, and M. Soljacic, Opt. Expr. 20, A366 (2012).

[8] S. Basu, Z. M. Zhang, and C. J. Fu, Int. J. En. Res. 33, 1203 (2009).

[9] G. S. Agarwal, Phys. Rev A 11, 253 (1975).

[10] W. Eckhardt, Z. Phys. B 46, 85 (1982).

[11] I. A. Dorofeyev and E. A. Vinogradov, Phys. Rep. 504, 75 (2011).

[12] A. V. Shchegrov, K. Joulain, R. Carminati, and J.-J. Greffet, Phys. Rev. Lett. 85, 1548 (2000).

[13] K. Joulain, R. Carminati, J.-P. Mulet, and J.-J. Greffet, Phys. Rev. B 68, 245405 (3003).

[14] Handbook of Optical Constants of Solids (Ed. E. Palik), Academic, New York 1998.

[15] P. Ben-Abdallah, K. Joulain, J. Drevillon, and G. Domingues, Appl. Phys. Lett. 94, 153117 (2009).

[16] R. Messina, J.-P. Hugonin, J.-J. Greffet, F. Marquier, Y. de Wilde, et al., Phys. Rev. B 87, 085421 (2013).

[17] Y. de Wilde, F. Formanek, R. Carminati, B. Gralak, P.-A. Lemoine, et al., Nature 444, 740 (2006).

[18] A. Babuty, K. Joulain, P.-O. Chapuis, J.-J. Greffet, and Y. D. Wilde, Phys. Rev. Lett. 110, 146103 (2013).

[19] A. Kittel, U. Wischnath, J. Welker, O. Huth, F. Rüting, et al., Appl. Phys. Lett. 93, 193109 (2008).

[20] F. Huth, M. Schnell, J. Wittborn, N. Ocelic, and R. Hillenbrand, Nat. Mat. 10, 352 (2011).

[21] A. C. Jones and M. B. Raschke, Nano Lett. 12, 1475 (2012).

[22] Q. Weng, S. Komiyama, L. Yang, Z. An, P. Chen, et al., Science 360, 775 (2018).

[23] S. Komiyama, J. Appl. Phys. 125, 010901 (2019).

[24] E. G. Cravalho, C. L. Tien, and R. P. Caren, J. Heat Transf. 89, 351 (1967).

[25] G. A. Domoto and C. L. Tien, J. Heat Transf. 92, 399 (1970).

[26] R. F. Boehm and C. L. Tien, J. Heat Transf. 92, 405 (1970).

[27] R. S. DiMatteo, Enhanced Semiconductor Carrier Generation via Microscale Radiative Transfer; MPC - An Electric Power Finance Instrument Policy; Interrelated Innovations in Emerg. ing Energy Technologies, Master's Thesis, Massachusetts Institute of Technology, Cambridge, MA 1996.

[28] M. D. Whale, A Fluctuational Electrodynamic Analysis of Microscale Radiative Transfer and the Design of Microscale Thermophotovoltaic Devices, Master's Thesis, Massachusetts Institute of Technology, Cambridge, MA 1996.

[29] J. Pan, Opt. Lett. 25, 369 (2000).

[30] M. D. Whale and E. G. Cravalho, IEEE Trans. Energy Convers. 17, 130 (2002).

[31] S. M. Rytov, Y. A. Kravtsov, and V. I. Tatarskii, Principles of Statistical Radiophysics, Vol. 3, Springer, New York 1989.
[32] R. S. DiMatteo, P. Greiff, S. L. Finberg, K. A. Young-Waithe, H. K. Choy, et al., Appl. Phys. Lett. 79, 1894 (2001).

[33] P. Ben-Abdallah and K. Joulain, Phys. Rev. B (R) 82, 121419 (2010).

[34] S.-A. Biehs, E. Rousseau, and J.-J. Greffet, Phys. Rev. Lett. 105, 234301 (2010).

[35] S.-A. Biehs, P. Ben-Abdallah, and F. S. S. Rosa, in: Nanoscale Radiative Heat Transfer and Its Applications, Infrared Radiation (Ed. V. Morozhenko), IntechOpen 2012. DOI: 10.5772/ 37484. Available from: https://www.intechopen.com/books/ infrared-radiation/nanoscale-radiative-heat-transfer-and-itsapplications.

[36] A. I. Volokitin and B. N. J. Persson, Rev. Mod. Phys. 79, 1291 (2007).

[37] J. Pendry, J. Phys. 11, 6621 (1999).

[38] O. D. Miller, S. G. Johnson, and A. W. Rodriguez, Phys. Rev. Lett. 115, 204302 (2015).

[39] H. Shim, L. Fan, S. G. Johnson, and O. D. Miller, Phys. Rev. X 9 , 011043 (2019).

[40] W. Jin, R. Messina, and A. W. Rodriguez, Opt. Exp. 25, 14746 (2017).

[41] E. G. Cravalho, G. A. Domoto, and C. L. Tien, Progr. Aeron. Astro. 21, 531 (1968).

[42] C. M Hargreaves, Phys. Lett. A 30, 491 (1969).

[43] G. A. Domoto, R. F. Boehm, and C. L. Tien, J. Heat Transf. 92, 412 (1970).

[44] R. Ottens, V. Quetschke, S. Wise, A. Alemi, R. Lundock, et al., preprint arXiv:1103.2389 (2011).

[45] L. Hu, A. Narayanaswamy, X. Chen, and G. Chen, Appl. Phys. Lett. 92, 133106 (2008).

[46] T. Kralik, P. Hanzelka, M. Zobac, V. Musilova, T. Fort, et al., Phys. Rev. Lett. 109, 224302 (2012).

[47] R. St-Gelais, B. Guha, L. Zhu, S. Fan, and M. Lipson, Nano Lett. 14, 6971 (2014).

[48] B. Song, Y. Ganjeh, S. Sadat, D. Thompson, A. Fiorino, et al., Nat. Nanotech. 10, 253 (2015).

[49] M. Lim, S. S. Lee, and B. J. Lee, Phys. Rev. B 91, 195136 (2015).

[50] K. Ito, A. Miura, H. lizuka, and H. Tosiyoshi, Appl. Phys. Lett. 106, 083504 (2015).

[51] J. I. Watjen, B. Zhao, and Z. M. Zhang, Appl. Phys. Lett. 109, 203112 (2016).

[52] B. Song, D. Thompson, A. Fiorino, Y. Ganjeh, P. Reddy, et al., Nat. Nanotech. 11, 509 (2016)

[53] M. P. Bernardi, D. Milovich, and M. Francoeur, Nat. Comm. 7, 12900 (2016).

[54] S. Lang, G. Sharma, S. Molesky, P. U. Kränzien, T. Jalas, et al., Sci. Rep. 7, 13916 (2017).

[55] M. Ghashami, H. Geng, T. Kim, N. Iacopino, S. K. Cho, et al., Phys. Rev. Lett. 120, 175901 (2018).

[56] M. Lim, J. Song, S. S. Lee, and B. J. Lee, Nat. Comm. 9, 4302 (2018).

[57] A. Fiorino, D. Thompson, L. Zhu, B. Song, P. Reddy, et al., Nano Lett. 18, 3711 (2018).

[58] A. Narayanaswamy and G. Chen, Appl. Phys. Lett. 82, 3544 (2003).

[59] A. R. Forouhi and I. Bloomer, Phys. Rev. B 38, 1865 (1988).

[60] R. Messina and P. Ben-Abdallah, Sci. Rep. 3, 1383 (2012).

[61] V. B. Svetovoy and G. Palasantzas, Phys. Rev. Applied 2, 034006 (2014). 
[62] K. Park, S. Basu, W. P. King, and Z. M. Zhang, JQSRT 109, 305 (2008).

[63] S. Basu and Z. M. Zhang, Appl. Phys. Lett. 95, 133104 (2009).

[64] M. P. Bernardi, O. Dupré, E. Blandre, P.-O. Chapuis, R. Vaillon, et al., Sci. Rep. 5, 11626 (2015).

[65] K. Chen, P. Santhanam, and S. Fan, Apl. Phys. Lett. 107, 091106 (2015).

[66] E. Blandre, P.-O. Chapuis, and R. Vaillon, Sci. Rep. 7, 15860 (2017).

[67] J. DeSutter, R. Vaillon, and M. Francoeur, Phys. Rev. Applied 8, 014030 (2017).

[68] R. Vaillon, J.-P. Perez, C. Lucchesi, D. Cakiroglu, P.-O. Chapuis, et al., Opt. Exp. 27, 347515 (2019)

[69] M. S. Mirmoosa and C. R. Simovski, Phot. Nanostruct. Fundam. Appl. 13, 20 (2015).

[70] I. S. Nefedov and C. Simovski, Phys. Rev. B 84, 195459 (2011).
[71] C. R. Simovski, S. Maslovski, I. Nefedov, and S. Tretyakov, Opt. Exp. 21, 14988 (2013).

[72] M. S. Mirmoosa, S.-A. Biehs, and C. R. Simovski, Phys. Rev. Appl. 8, 054020 (2017).

[73] S. Jin, M. Lim, S. S. Lee, and B. J. Lee, Opt. Exp. 24, A635 (2016).

[74] S.-A. Biehs, M. Tschikin, and P. Ben-Abdallah, Phys. Rev. Lett. 109, 104301 (2012).

[75] S.-A. Biehs, S. Lang, A. Yu. Petrov, M. Eich, and P. BenAbdallah, Phys. Rev. Lett. 115, 174301 (2015).

[76] B. Liu and S. Shen, Phys. Rev. B 87, 115403 (2013).

[77] S. Lang, M. Tschikin, S.-A. Biehs, A. Yu. Petrov, et al., Appl. Phys. Lett. 104, 121903 (2014).

[78] M. Tschikin, S.-A. Biehs, P. Ben-Abdallah, S. Lang, A. Yu. Petrov, and M. Eich, JQSRT 158, 17 (2015).

[79] A. Fiorino, L. Zhu, D. Thompson, R. Mittapally, P. Reddy, et al., Nat. Nanotechn. 13, 806 (2018). 